\title{
Cooling Process of Gas Turbine Blade: A Comparison Study
}

\author{
Akram Luabi ${ }^{a, b^{*}}$, Naseer Hamza ${ }^{a}$ \\ ${ }^{a}$ Mechanical Engineering Department, University of Al-Qadisiyah, Ad'Diwaniyah 58001, Iraq \\ ${ }^{b}$ Al-Diwaniyah Gas Turbine Power Plant, Ad'Diwaniyah 58001, Iraq
}

\section{ARTICLE INFO}

\section{Article history:}

Received 02 June 2020

Received in revised form 10 July 2020

Accepted 20 July 2020

\section{Keywords:}

Gas turbine

Blade

Film cooling

Internal cooling

\begin{abstract}
A B S T R A C T
The gas turbine engines are occupied an important sector in the energy production and aviation industry and this important increase day after day for their features. One of the most important parameters that limit the gas turbine engine power output is the turbine inlet temperature. The higher is the turbine inlet temperature, the higher is the power output or thrust but this increases of risks of blade thermal failure due to metallurgical limits. Thus the need for a good and efficient process of blade cooling can lead to the best compromise between a powerful engine and safe operation. There are two major methods: film or external cooling and internal cooling inside the blade itself. . In the past number of years there has been considerable progress in turbine cooling research and this paper is limited to review a few selected publications to reflect recent development in turbine blade film cooling. The maximum drop in the surface temperature of the gas turbine blade and associated thermal stress - due to incorporating cooling systems- were $735{ }^{\circ} \mathrm{C}, 1217 \mathrm{~N} / \mathrm{mm}^{2}$ respectively.
\end{abstract}

() 2020 University of Al-Qadisiyah. All rights reserved.

\section{Introduction}

The experimental, theoretical, and computational in the recent different investigations relevant to this work were presented such as the effect of raise the number of cooling duct and the coupled heat transfer (conjugate) of gas turbine bucket. Literature scanning mentions that conjugate heat transfer CHT examination performs an important function to guide the temperature distributions for the heat transfer representations.

The turbine inlet temperature is the most important parameter that influences the thermal efficiency of the power plants. Gas turbines are run at high inlet temperatures to satisfy the requirements of the power plants like the improved thermal efficiency and increasing the production of the energy. Innovative internal and external cooling methods are introduced for the new gas turbine engines to cool the blades and avoid material failure at extreme temperatures. Improved cooling techniques will allow the turbine operation to be closer and closer to the maximum permissible temperature for combustion and thus meet the power output requirement, especially at peak load. The cooling systems in the gas turbine blade are very complicated even that some times each application has its cooling scheme. Fig. 1 show the cooling passages inside the blade. The mathematical model of cooling systems is based on the forced convection heat transfer equations majorly and related formulas.

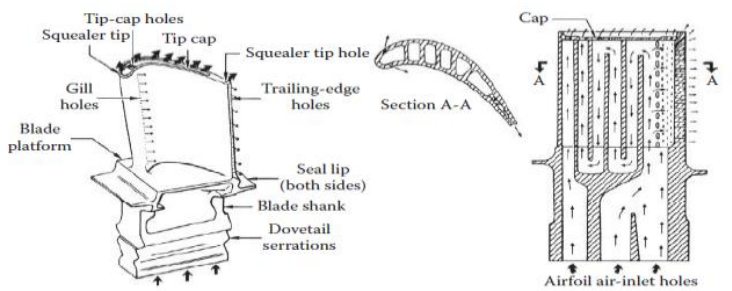

Figure 1. Stage-1 high-pressure turbine rotor blade for the GE CF6 engine. [1]

* Corresponding author.

E-mail address: akramalkhanfar@gmail.com (akram Luaibi) 
There are many surveys done to scan the outcomes of researchers in the field of gas turbine technology and to continue this effort, this study aims to compare recent studies especially to cover recent last years.

\section{Analysis of turbine blade cooling mechanisms}

In the brief review below some of the pertinent work available in the open literature. At the summary review below some of the relevant study which available in the modern literature.

\subsection{External cooling (Film cooling)}

Film cooling is used to protect a solid surface from a high temperature of the mainstream by releasing a coolant on the surface. This secondary fluid can be considered as a heat sink for the heat flow from the hot mainstream or as an insulating layer between the hot mainstream and the surface.

Few researchers performed experimental work for instance many references cited the work of Luckey et. al. [2] empirical examination is used to simulate the airfoil profile of leading edge by using the stagnation zone of a cylinder mounted in a cross-flow a cylindrical with some of film cooling lines. They associated their outcomes for the optimal value of the blowing ratio and insertion angle. Experiments were fulfilled with an individual row of spanwise angled cooling channels for a domain of the coolant blowing ratio with independent flow-to-wall temperature.

The local heat flux data measurements are offered for injection angles of cooling channel at $25^{\circ}, 35^{\circ}$, and $45^{\circ}$ with the row of passages located at three sites comparative to the stagnation line on the drum. Results see that path of (channel-to-channel) variations of heat flux shorthand due to film cooling and indicate the optimum performance for film cooling conditions Mehendale et. al. [3].

Yao et al. [4] carried out an experimental investigation to study the performance of the cooling film for a consoles of single row set on a curved plate model in order to simulate the suction side of the blade. Numerical simulation was accomplished in order to disclose the detailed feature of the consoles film cooling (consoles on a curved surface). The results were compared to a mode of cylindrical holes. Martini et al. [5] studied the heat transfer and the cooling performance of the film of the cutback of the gas turbine airfoil trailing edge by using IR thermography method for different forms of internal cooling. The internal design effect on the cooling effect for the ejection slots was studied as well. The results showed that the shedding of the vortex from the side lip pressure was the main reason beyond the degradation in the cooling effectiveness of the film.

Je-Chin Han et al. [6] performed the experiments in three zones of a stator blade of the gas turbine which are blade platform, span, and tip. The blade platform and span investigations were done on the rotor blade of the high pressure turbine in median flow situations. Film-cooling performance or degree of cooling was appreciated in terms of cooling duct form, blowing ratio, and mainstream to coolant density ratio. The blade crest study was carried out in a blow-down flow turn in a transonic flow medium. The cooling degree was valued in terms of tip clearance and blowing ratio. Confined heat transfer coefficient evaluations were also performed. Mainstream pressure wastage was measured for blade platform and tip filmcooling. Results showed that the blade platform cooling demands a collection of purge flow at upstream and discrete film-cooling channels downstream to cool the total platform.
The formed cooling passages provided that vast film covering and higher film-cooling performance than the circular passages whereas also producing minimum mainstream pressure losses.

Colban et al. [7] performed the film-cooling effectiveness of cylindrical channels and compared them with the vane-formed ducts on a turbine blade end wall. They had several experimental data for setup closes to that as the present work, with two passages having the same layout, one with circular holes and the other with vane-formed ducts. The missing aspect in the research that has been done in the examination into the effect of variance channels configuration and form on the blade cooling of the gas turbine and comparing the result in obvious parameters. Yang et al. [8] anticipated the cooling effectiveness of the film and the coefficient of heat transfer for three different types of the arrangement of the film and the hole. In the first one, the holes were designed to be located on the tips mid-camper line. In the second, the holes were created to be set in the stream of leakage flow (on the tip) and the region of heat transfer. While the third arrangement was the combination between the first and the second, respectively. The results of this study showed that the second configuration provided better performance than the others.

Acharya et al. [9] presented that the cooling injection of the film decreased the ratio of the local pressure and changed the leakage vortex nature. High adiabatic effectiveness of the film and low coefficient of heat transfer were noticed lengthways the trajectory of the coolant with very small values of the cooling jet cross spreading. Better efficiency of the downstream of the coolant might be provided by increasing the gap of the tip. However, at the smallest gap, the coolant directly impinged on the shroud surface resulting in a high effectiveness at the point of impingement.

Hohlfeld et al. [10] presented a numerical study about losses of the cooling flow and the microcircuit channels. The advantages of the cooling flow of the external film that resulted from using microcircuit was presented. The results predicted that the microcircuit exits blowing and the cooling of the mid chord were independent. Besides, for all modes of the cooling of the microcircuit film presented in this study, aside vortex of the pressure was developed. In addition, good cooling effectiveness was obtained from the exiting of the holes of the dirt purge when the tip gap was small. However, low cooling effectiveness was observed for the larger gap of the tip.

Goldstein R. J et. al. [11] presented the cooling of the film by introducing fluid (secondary) along the high temperature surface in order to protect these surfaces. Ito et al. [12] presented a film cooling effect on the blade of a gas turbine by calculating the cooling effectiveness of the film holes for the surfaces of different convexities and curvatures. Kumar et. al. [13] presented a detailed investigation about film cooling of the blades of a gas turbine. ANSYS WORKBENCH V. 15.0 software was used for the numerical simulation and solving the governing differential equations. Effect of using different configurations of the holes that changes the cooling effectiveness was also investigated. The results of this study showed that the conclusions presented by other researchers about using cooling channels were correct reasonable. In addition, the increasing in cooling efficiency due to using cooling channels was due to heat transfer enhancement. Increasing the flow rate of the mass was a good factor for increasing cooling effectiveness (an optimal mass flow rate was required).

\subsection{Internal cooling (cooling holes)}

Khari brahmaiah et.al. [14] discuss the four various models to analyze the heat transfer through a gas turbine blade, blade is analyzed without and with channels that have different hole numbers $(5,9 \& 13)$. According to this 
study, the temperature distribution and transfer rate for the blade with 13 holes are optimal.

Structural analysis is done by the ANSYS software with blade at different materials, Inconel-718 and Chromium steel. The thermal properties of Inconel-718 material are better whereas induced stresses are lesser as compared to the Chromium steel.

Josin et al. [15] showed a comprehensive study and design of the cooling passages of the blades of a gas turbine. The geometrical modeling was created by CATIA V.5. Both thermal and structural analyses were performed by using ANSYS 14.0 finite element software which is commercially available in its student edition. GDT-11, U-500, and IN-738 alloy were considered as the material that used in this study. The first step of the analysis was to choose the material that shows better results for the first stage of the turbine blades. The next step was the modeling of the blade by modifying its geometry into a serpentine model in addition to increase the number of the hole. Temperature distribution from the thermal analysis and maximum elongation from the structural analysis were calculated and observed at the tip and the root sections of the blade, respectively. The comparisons between the three materials used in this study showed that GTD-11 offered the maximum deformation, maximum stresses, and could withstand higher temperatures around $8570 \mathrm{C}^{\circ}$ without any damage to the blade because of the higher melting temperature.

Öztekin et al. [16] observed that the numerical results are in perfect coincidence with experimental results when developed wall functions and the model of $k-\varepsilon$ turbulence in the principle of local alteration of the pressure coefficient. Maximizing the dimensionless blade-to surface space produced deficiency the coefficient of pressure $(\mathrm{Cp})$ at the stagnation end. B.H. Dennis et al. [17] presented an optimized design for the cooling passage of a gas turbine blades by using the reduced method of conjugate heat transfer. The compressor last stage in the gas turbine presented in this study provided the cooling air. It was noted that the shape of the cooling channels and its location affected the heat transfer amount, temperature gradient inside the blades, temperature distribution, and cooling air-mass flux. Air was designed to flow inside the blades from its root and leave from its tip or trailing and leading edges.

$\mathrm{Ni}$ et al. [18] simulated a methodology and an analysis of heat transfer and applied it for studying the cooling process of an air force film of a turbine van with 648 holes. The results of this work showed that air flows from the holes (cold air) created a protective layer all around the surface of the airfoil and the end walls. Lower temperature values were noticed on the entrance to the holes as well as the surfaces that were subjected to a high temperature hot gas. Choo et al. [19] tested the micro-scale jet impingement on a heated flat plate and estimated the characteristics method of heat transfer by using correlation equations with experimental data on average and local Nu number in terms of Reynolds number and blade-toplate distance. Numerical study, using Reynolds-averaged Navier-Stokes (RANS), coupled with the k- $\varepsilon$ model has been appointed as an important norm design and the simulation analysis. B. Deepanraj et al. [20] used a numerical analysis thermal effectiveness due to loading status. TitaniumAluminum alloy properties and variant cases with different hole numbers of $(7,8,9,10,11$, and 12) were performed. Thermal stress, temperature distribution, and deflection were obtained using ANSYS software for several number of channels. The results explained that the decrease in the temperature values and the reduction in the temperature drop was motivated by the increasing number of the blade channels. Reddy et al. [21] has tested the stainless steel thermal and structural analysis. Four variant cases containing of blade without and with different number of passages (7, 8, 9, and 10) were examined to discover the perfect cooling channels number. It is found that as the number of cooling channels increases the temperature distribution rise up. The SOLID WORKS software is used to simulate the structural analysis.

The results showed that the blade with 10 № of holes is more stresses than the other models. Lastly, the model with nine holes has produced the optimum solution. A.B. Moskalenkoa et al. [22] the numerical calculations were carried out using CFD based on the FEM. The coefficient of heat transfer and heat distribution of the bucket from the cooling domain to the cooling hole wall were selected as the standard of cooling efficiency.

Abdulla R. Al Ali et al. [23] was fulfilled the numerical analysis of different heat transfer methods of jet impingement on a half-circular face. The comparison of these cases was based on the effective heat transfer by obtaining top Nusselt number and minimum surface temperature as heat transfer by convection is becoming the dominant situation and by compared between central and side jet configuration based on average Nusselt number at different Reynold numbers. The results were obtained to be in convention with the experimental investigation. For more highlight physics of the flow, a sensibility test on the jet impingement configuration and flow status were specified and was demonstrated to the internal cooling of the first stage bucket. HARSHA D A et al. [24] investigated the effects of material on thermal and structural analysis of blade designed with 12 holes as shown in Fig. 2. He compared the Titanium alloy with aluminum and observed that the total rate of heat transfer and the leading edge temperature were maximum and minimum for the 12 holes blade, respectively.

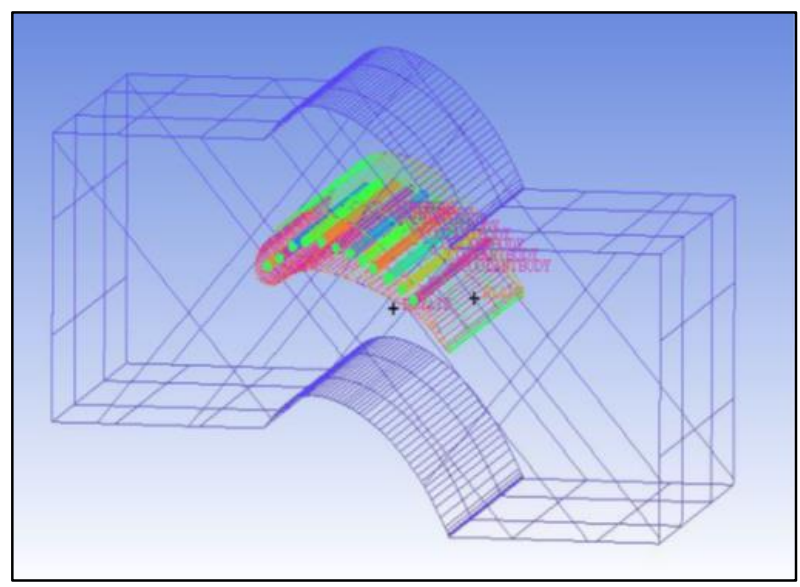

Figure 2. Geometry model with boundary walls [24].

Soo-Yong Cho. et al. [25] investigated the conjugate Heat transfer on a blade of a gas turbine for various cases without and with different number of cooling channels. It was noted that the temperature of the leading edge was minimum while the overall heat transfer rate was higher for the blade within 13 holes. The analysis was examined for two alloy constructions, Inconel 718 and Chromium steel. The results showed the maximum value of the heat flux for the Inconel 718 blade with 13 channels and the thermal stresses were under allowable limits. K. Mazaheri et al. [26] examined the optimization of the configuration of the blade cooling holes. The goal was maximization of the temperature distribution. The conjugate heat transfer technique was applied to perform the analysis. The internal and external heat transfer was performed in a central scheme to find out the optimal function. Results showed that the method of temperature gradient in moving the fourth hole a far from the trailing edge and also reducing the 
volume of all holes, while the criterion of maximum temperature results maximize in their volume.

A. Ziaei-Asl et al. [27] studied the effects of varying the thickness of a Thermal Barrier Coating (TBC) on the style of temperature distribution for a turbine blade. The blade was modeled to contain several cooling channels to increase the cooling efficiency. Several micro-passage were designed to be located on the blade tip in order to take out the coolant. The TBC is considered as an elastic material and the elastic modulus was computed from the stress-strain curve. A.Hasanpour et al. [28] solved the conjugate heat transfer of a metal blade designed in four different hole shapes and configurations. Four cylindrical holes in shape with k- $\varepsilon$ (RKE) for turbulence modeling were used in this work.

Couch. et. al. [29] examined a novel technique for cooling called a microcircuit technique. This technique combines the injection of the pressure side and the internal convection on the tips of the turbine blades. The dirt purge holes were designed to be on the tip to expel the dirt from the blade. In order to check the effectiveness of the holes on the tip along with the suggested microcircuit technique, the design was tested experimentally using wind tunnel tests. Both blowing ratio and gap size of the tip were variables for different cooling configurations. From the results of this study, a significant cooling increasing was provided by the dirt purge holes. The injected coolant out from these holes affected the shroud and flooded the tips that resulted in reducing the leakage of the tip flow.

Chandrakant et al. [30] conducted a comparison study between the turbulator and using a helicoidally ducted blade for several geometrical proportions to estimate which is better on cooling. It was noted a significant enhancement for the characteristics of cooling of the turbine blade when using turbulator geometry of the larger ratio of the radial thickness of the turbulator to the helicoidally duct outer diameter (e/D). Htwe et al. [31] Gas turbines have an indispensable part in electric power generation. Gas turbine innovation is utilized as a part of a ranking of arrangements for electric power epoch. The leading and trailing edge of the turbine rotor is the most critical segment in the gas turbine power plant. John et al. [32] designed and analyzed a gas turbine blade. CATIA and ANSYS were used for the design and the finite element analysis, respectively. Life assessment and post processing were included in this work as well. ANSYS milieu facilities were used for the complex mesh generation and application of the working and boundary conditions.

Han et al. [33] turned the light on the techniques used for the blade cooling, such as rib-turbulated cooling, pin-fin cooling, and jetimpingement for two cases, rotating and fixed blades. Priyanka Singh et al. [34] used numerical analysis to study the heat transfer of gas turbines for six completely different models containing $(5,9,13)$ in a single line row of passages and compared with another model that has $(9,13)$ holes set in the 3 row. They developed a new model with 14 passages in the staggered arrangement. They found that the temperature gradient on the 13 passages was consistently distributed along the surfaces of the blade compared to 13 single line row. Heat transfer increased in the arrangements of 13 and 14 staggered holes.

Allen and Kofskey et al. [35] performed some visualization tests to see what these secondary flows looked like. Additionally, they also studied the effect of ejecting flow from the turbine tips on the shape of the secondary flows. At the time of these tests, engine temperatures were not so high as to require cooling of the turbine blades for operation. The cooling of the turbine blade could be considered as a necessary aspect of engine design. The cooling method of the rotor blade of the turbine by air passing inside the hollow blades and taking out it from the tip of the blade can be The results of the reviewed previous researches were summed up in table 1. considered one of the most practical ways of varying the pattern of the secondary flow.

G. Narendranath et al. [36] analyzed the 1st stage of the rotating gas turbine blade using ANSYS 9.0. The blade material used in the analysis is N155 alloy. The structural and thermal analyses were conducted numerically (FEM). The temperature gradient from LE to the TE on the surface of the blade was ranged from $839.531{ }^{\circ} \mathrm{C}$ to $735.162{ }^{\circ} \mathrm{C}$ at the blade tip. It is noticed that the minimum thermal stress was 1450 which is less than the yield strength of the material and the maximum thermal fatigue was 1217

V.Vijaya Kumar et al. [37] investigated a specific power turbine design in order to maximize the turbojet engine. For an obvious awareness of the compound thermal and mechanical stresses for the centrifugal and axial mechanical forces. The circumferential rotor speed and stream velocities were preserved in the feasible range to reduce the losses. The base profiles are completed using the potential flow method taking into consideration all flow conditions during the analytical solution process.

The last stage of the compressor provides the cooling air for the blade holes. The configurations of the interior cooling holes influence the amount of transferred heat rate and the distribution of the temperature at the interior of the blade; besides that, the cooling air mass flow rate will affect the total turbine efficiency. The trivial choice for holes or passages is a circular shape, and the coolant fluid enters the blade hub and then comes out from its tip or the trailing edge. In the modern design of blades, the flow inside the adjacent holes takes various orientations B.H. Dennis et al [38].

Dulikravich et al. [39, 40] applied these optimization algorithms for analyzing the aerothermal -elastic characteristics of the blade of the turbine.

The channels were split using ribs and their thicknesses and locations were specified according to a genetic algorithm. A restriction in the research development scope is appeared by using this model. Another study Kennon et. al. [40] investigated the optimized number, location, and size of the circular channels that were placed near the surface of the blade. The number of channels was limited by using the upper and lower limits of the channel diameter for minimizing the total transferred heat.

Verstraete et al. [41, 42] optimized the position and size of the five channels having circular shapes in the 3D blade. Maximizing the blade lifetime and limiting the flux of the cooling air mass are the main objectives of the study.

Namgoong et al. [43] utilized alternative models and experiment optimization design methods for reducing the loss in the pressure for the 3D U-bend duct having 4-edges. Two edges were fixed, while the remaining two edges were permitted to change at 8-points within the channel. A recent study by Nagaiah et al. [44] used the quadrilateral channel with an internal cooling system represented by the tabulating the ribs of channel walls. A semi-cylinder shape of ribs was adopted. The external flow on and close to the blade was not considered. A multiobjective optimization was adopted for increasing the convection coefficient of the transferred heat, the effective surface of heat transfer, and decreasing the loss in the pressure of the channel pressure.

The main emphasis of the previous studies is on the predefined channels. This can remove from our research scope many optimal shapes.

Nowak et al. [45] optimized the maximum thermal gradient and temperature using the genetic algorithm method with four curves of Bezier. Some controlling points were constrained for decreasing the design variable numbers. This led to losing the smoothness control on the surface channel, in which many unsmooth corners and shapes were included. 
Table 1. Summary of results of significant literature studies.

\begin{tabular}{|c|c|c|}
\hline Author & Year & Method \\
\hline Yang et al [8] & 2004 & $\begin{array}{l}\text { Three different types of the arrangement of the } \\
\text { film cooling }\end{array}$ \\
\hline G. Narendranath et.al [36] & 2006 & $\begin{array}{l}\text { Analyzed the 1st stage of the rotating gas } \\
\text { turbine blade using Ansys } 9.0 \text { by used n } 155 \\
\text { alloy }\end{array}$ \\
\hline Choo et al [19] & 2009 & $\begin{array}{l}\text { Using correlation equations with experimental } \\
\text { data of nu number. }\end{array}$ \\
\hline
\end{tabular}

Ni et al. [18]

Apply the cooling process of an air force film of a turbine van with 648 holes

Used titanium- aluminum alloy properties and

B. Deepanraj et.al [20]

2011 variant cases with different hole numbers of (7, $8,9,10,11$, and 12)

Discuss the four various models without and with channels that have different hole numbers

K hari brahmaiah et.al [14]

Reddy [21]

Kumar [13]

B.h. Dennis [17]

Abdulla r. Al ali et al [23]

Moskalenkoa et al [22]

2016

K. Mazaheri et al [26]

2016

A. Ziaei-asl et al [27]
(5, 9\&13) with different materials, Inconel-

718 , and chromium steel.

Tested the stainless steel thermal and structural analysis. Four variant cases containing of blade without and with different number of passages (7, 8, 9, and 10)

Used for the numerical simulation and solving the governing differential equations

Using the reduced method of conjugate heat 2015 transfer

Numerical analysis of different heat transfer methods of jet impingement on a half-circular face.

Comparison of coolant air and steam was carried out

The internal and external heat transfer was performed in a central scheme to find out the optimal function

Studied the effects of varying the thickness of 2017 a thermal barrier coating (TBC) on the style of temperature distribution for a turbine blade
Results

The second configuration provided better performance than the others.

The temperature gradient from LE to the TE was ranged from $839^{\circ} \mathrm{c}$ to $735^{\circ} \mathrm{c}$ at the blade tip. The minimum thermal stress was $1450 \mathrm{~N} / \mathrm{mm}^{2}$ which is less than the yield strength and the maximum thermal fatigue was $1217 \mathrm{~N} / \mathrm{mm}^{2}$.

Using (rans), coupled with the $\mathrm{k}-\varepsilon$ model has been appointed as an important norm design and the simulation analysis. Air flows from the holes (cold air) created a protective layer all around the surface of the airfoil and the end walls. Lower temperature values were noticed on the entrance to the holes as well as the surfaces that were subjected to a high temperature hot gas.

The decrease in the temperature values and the reduction in the temperature drop was motivated by the increasing number of the blade channels.

The blade with 13 holes is optimal and the thermal properties of Inconel-718 material are better whereas induced stresses are lesser as compared to the chromium steel.

Blade with 10 № of holes is more stresses than the other models. Lastly, the model with nine holes has produced the optimum solution.

Increasing the flow rate of the mass was a good factor for increasing cooling effectiveness

The shape of the cooling channels and its location affected the heat transfer amount, temperature gradient inside the blades, temperature distribution, and cooling air-mass flux.

A sensibility test on the jet impingement configuration and flow status was specified and was demonstrated to the internal cooling of the first stage bucket.

Coolant steam is more efficient than the air when examined using the same conditions.

The location and the form of the last hole showed a significant role for all goal functions.

The coating thickness has a critical impact on the thermal and mechanical characteristics of the blade besides increasing the working period of the blade (blade life).

\section{Discussion of Analysis Results}

During the last decade, the studies of optimization were significantly advanced. The optimization algorithms are now used broadly especially in engineering applications due to the development in the computational hardware that used the numerical methods in solving the problems. The cooling system of the turbine blade is one of the applications that could be modeled and analysed using these algorithms. The result of Titus.R et. al. [15] showed that increasing holes number with reducing its size and reducing the gap between them led to a good design. The existing design and the modified design (increasing the number of the holes) showed
$1250 \mathrm{~K}$ and $1191.6 \mathrm{~K}$, respectively. However, the serpentine design is cost effective and requires more effort because of the difficulties in the manufacturing processes compared to the other available designs.

Results of Moskalenko et al. [22] showed the comparison of coolant air and steam was carried out, and the computations were done using ANSYS Fluent tool. The coolant steam is more efficient than the air when examined using the same conditions as shown in Fig. 3 . 


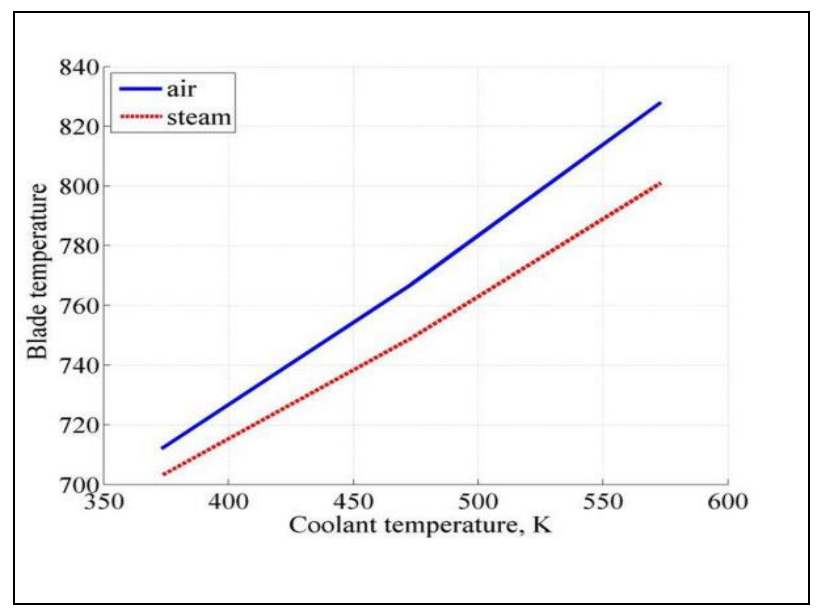

Figure 3. Shows the result of A.B. Moskalenkoa et al. [22].

Results of H.D.A et. al. [24] showed that, for all materials used in the blade modeling, the Nusselt number and the coefficient of the heat transfer were constant at the surfaces of the hole while the coefficient of the heat transfer was high at the entrance as shown in Fig. 3.

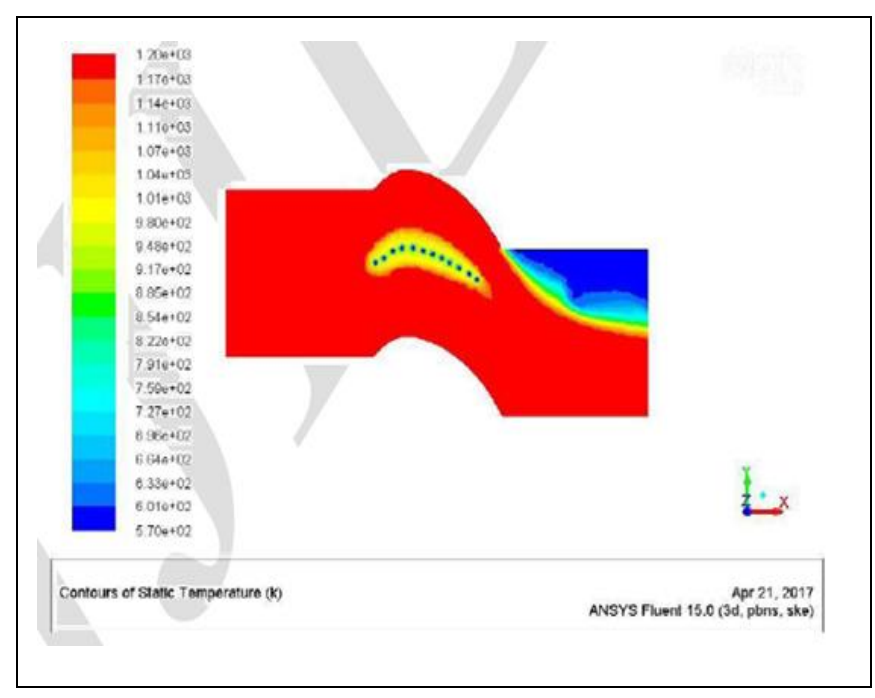

Figure 4. Shows the result of HARSHA D A et al. [24].

The results of Ziaei-Asl et. al. [27] temperature distribution, thermal stress, and thermal strain showed that the coating thickness has a critical impact on the thermal and mechanical characteristics of the blade besides increasing the working period of the blade (blade life) as shown in Fig. 5.

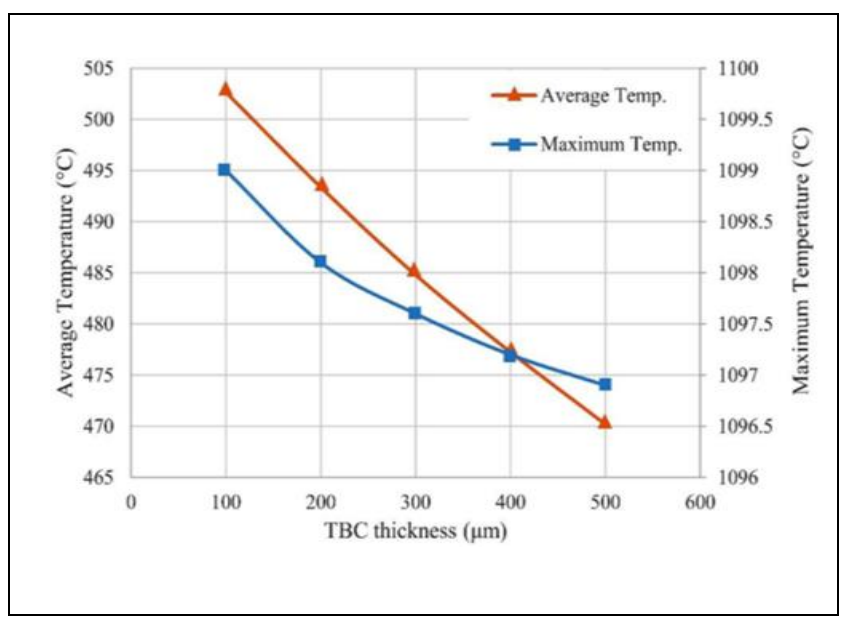

Figure 5. Average and maximum temperature vs. the TBC thickness

[27].

The result of Narendranath et. al. [36] noticed that the temperature gradient from LE to the TE on the surface of the blade was ranged from $839.531{ }^{\circ} \mathrm{C}$ to $735.162{ }^{\circ} \mathrm{C}$ at the blade tip. The minimum thermal stress was 1450 which is less than the yield strength of the material and the maximum thermal fatigue was 1217.

And the results of Hasanpour et. al. [28] showed that temperature distribution at the mid-span of the external surface of the blade was in a good agreement compared to the experimental data as shown in Fig. 6. The maximum value of the temperature was noticed at the trailing edge of the van right at the very thin metal of the van. This value of the temperature was closer to the passage free stream temperature than that corresponding of the coolant, because of thermal resistance which was greater than the external conduction or convection resistance within the metal.

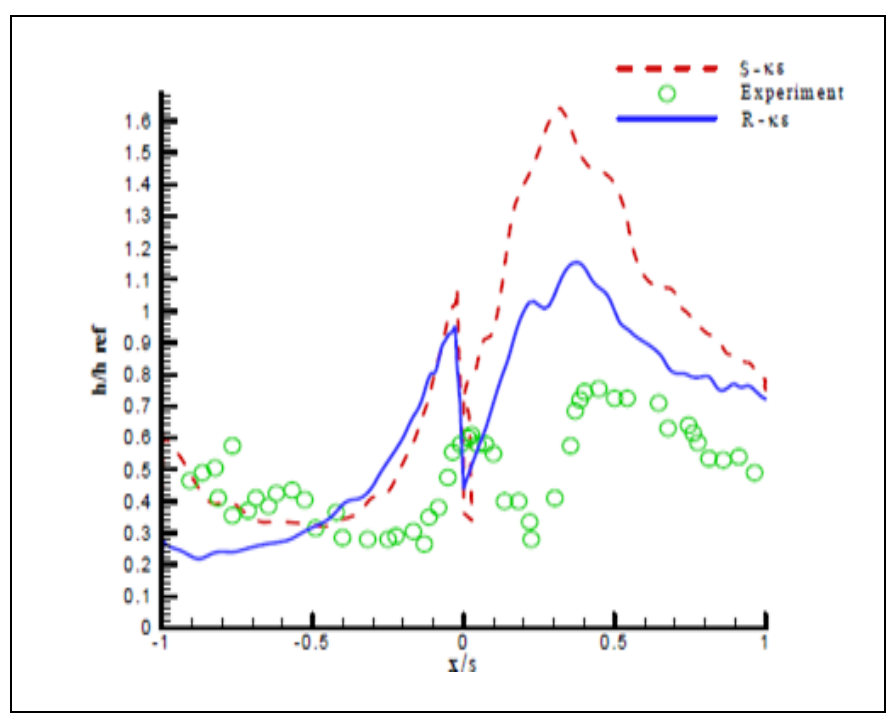

Figure 6. Experimental and calculated data of [28]. 


\section{Conclusions}

The usage of a cooling process in the gas turbine blade is essential. It may be an external or internal cooling according to the area of application. Some of application like aviation engines require very high turbine inlet temperature and thus usage either external or internal is not sufficient and need to incorporate both of them. Some applications need only internal cooling like in medium size gas turbine used in electric power plants. The interest in the development of internal cooling increased considerably in the last decade due to its efficient merits in minimizing hot spots that occurred due to high turbine inlet temperature. In addition, some researchers are interested to investigate the effect of adding ribs inside the serpentine passages of cooling holes and the optimum distribution of them. The selection of appropriate method is a matter of economic justification according to the area of application.

The goal of previous studies aforementioned is to minimize the hot spots that may occur due to severe conditions and resulted in thermal stresses as stated in Table 1. This goal is achieved via different approaches as stated in this comparison study and the great achievement belongs to Narendranath et. al. [36] where the maximum temperature is lowered by $\mathbf{1 2} \%$ and thermal stress was lowered by $16 \%$.

\section{REFERENCES}

[1] J.-C. Han, S. Dutta, S. Ekkad, Gas turbine heat transfer and cooling technology, CRC press, 2012.

[2] D. Luckey, D. Winstanley, G. Hanus, M. L'Ecuyer, Stagnation region gas film cooling for turbine blade leading-edge applications, Journal of Aircraft, 14(5) (1977) 494-501.

[3] A. Mehendale, J. Han, Influence of high mainstream turbulence on leading edge film cooling heat transfer, (1992).

[4] Y. Yao, J.-Z. Zhang, L.-P. Wang, Film cooling on a gas turbine blade suction side with converging slot-hole, International journal of thermal sciences, 65 (2013) 267-279.

[5] P. Martini, A. Schulz, H.-J. Bauer, Film cooling effectiveness and heat transfer on the trailing edge cutback of gas turbine airfoils with various internal cooling designs, (2006).

[6] Z. Gao, D. Narzary, J.-C. Han, Turbine blade platform film cooling with typical stator-rotor purge flow and discrete-hole film cooling, Journal of Turbomachinery, 131(4) (2009).

[7] W. Colban, K. Thole, Influence of hole shape on the performance of a turbine vane endwall film-cooling scheme, International journal of heat and fluid flow, 28(3) (2007) 341-356.

[8] H. Yang, H.-C. Chen, J.-C. Han, Numerical prediction of film cooling and heat transfer with different film-hole arrangements on the plane and squealer tip of a gas turbine blade, in: ASME Turbo Expo 2004: Power for Land, Sea, and Air, American Society of Mechanical Engineers Digital Collection, 2004, pp. 177-187.

[9] H. Nasir, S.V. Ekkad, S. Acharya, Flat surface film cooling from cylindrical holes with discrete tabs, Journal of thermophysics and heat transfer, 17(3) (2003) 304-312.

[10] E.M. Hohlfeld, Film Cooling Predictions Along the Tip and Platform of a Turbine Blade, Virginia Tech, 2003.

[11] V. Eriksen, R. Goldstein, Heat transfer and film cooling following injection through inclined circular tubes, (1974).

[12] S. Ito, R. Goldstein, E. Eckert, Film cooling of a gas turbine blade, (1978).

[13] S. Kumar, CFD Analysis of Film Cooling in Gas Tusrbine Blade, 2015.

[14] K.H. Brahmaiah, M.L. Kumar, Heat transfer analysis of gas turbine blade through cooling holes, International Journal of Computational Engineering Research, ISSN, (2014) 2250-3005.

[15] J.G.a. Titus.R, Design Analysis First Stag Gas Turbine Blade With modification Cooling Passage Using Ansys, International Journal Latest Trends Eng. Technology (IJLTET), 3(4) (2014) 313.
[16] E. Öztekin, O. Aydin, M. Avcı, Hydrodynamics of a turbulent slot jet flow impinging on a concave surface, International communications in heat and mass transfer, 39(10) (2012) 1631-1638.

[17] B.H. Dennis, G.S. Dulikravich, I.N. Egorov, S. Yoshimura, D. Herceg, Three-dimensional parametric shape optimization using parallel computers, Computational Fluid Dynamics Journal, 17(4) (2009) 256-266.

[18] R.H. Ni, W. Humber, G. Fan, P.D. Johnson, J. Downs, J. Clark, P. Koch, Conjugate heat transfer analysis for a film-cooled turbine vane, in: ASME 2011 Turbo Expo: Turbine Technical Conference and Exposition, American Society of Mechanical Engineers Digital Collection, 2011, pp. 423-434.

[19] K.S. Choo, Y.J. Youn, S.J. Kim, D.H. Lee, Heat transfer characteristics of a micro-scale impinging slot jet, International journal of heat and mass transfer, 52(13-14) (2009) 3169-3175.

[20] B. Deepanraj, P. Lawrence, G. Sankaranarayanan, Theoretical analysis of gas turbine blade by finite element method, Scientific world, 9(9) (2011) 29-33.

[21] R. Reddy, THERMAL STRUCTURAL ANALYSIS OF GAS TURBINE BLADE USING SOLIDWORKS.

[22] A. Moskalenko, A. Kozhevnikov, Estimation of gas turbine blades cooling efficiency, Procedia Engineering, 150(1) (2016) 61-67.

[23] A.R. Al Ali, I. Janajreh, Numerical simulation of turbine blade cooling via jet impingement, Energy Procedia, 75 (2015) 3220-3229.

[24] H.D.A. . Cfd analysis on radial cooling of gas turbine blade, Novateur publications international journal of innovations in engineering research and technology [IJIERT],, 4 (2017).

[25] S.-Y. Cho, E.-S. Yoon, B.-S. Choi, A Study on an axial-type 2-D turbine blade shape for reducing the blade profile loss, KSME International Journal, 16(8) (2002) 1154-1164.

[26] K. Mazaheri, M. Zeinalpour, H. Bokaei, Turbine blade cooling passages optimization using reduced conjugate heat transfer methodology, Applied Thermal Engineering, 103 (2016) 1228-1236.

[27] A. Ziaei-Asl, M.T. Ramezanlou, EFFECTS OF THERMAL BARRIER COATING (TBC) THICKNESS ON TEMPERATURE DISTRIBUTION OF GAS TURBINE BLADE.

[28] A. Hasanpour, M. Farhadi, H. Ashorynejad, Hole configuration effect on turbine blade cooling, World Academy of Science, Engineering and Technology, 49(1) (2011) 240-244.

[29] E.L. Couch, Measurements of Cooling Effectiveness Along the Tip of a Turbine Blade, Virginia Tech, 2003.

[30] C.R. Kini, S.B. Shenoy, Y.N. Sharma, Computational conjugate heat transfer analysis of hp stage turbine blade cooling: effect of turbulator geometry in helicoidal cooling duct, World Academy of Science, Engineering and Technology, 2012(70) (2012) 645-652.

[31] H.H.W. Win Lai Htwe, Nyein Aye San, ,, Design and Thermal Analysis of Gas Turbine Blade, International Journal of Mechanical and Production Engineering,, 3(7) (2015).

[32] T.R. John.V, The Design and Analysis of Gas Turbine Blade, International Journal of Advanced Research and Studies,, 2(1) (Dec 2012).

[33] J.-C. Han, H.-C. Chen, Turbine blade internal cooling passages with rib turbulators, Journal of Propulsion and Power, 22(2) (2006) 226-248.

[34] P. Singh, O. Shukla, Heat transfer analysis of gas turbine rotor blade through staggered holes using CFD.

[35] H.W. Allen, M.G. Kofskey, Visualization Study of Secondary Flows in Turbine Rotor Tip Regions, (1955).

[36] G. Narendranath, S. Suresh, THERMAL Analysis Of A Gas Turbine Rotor Blade By Using Ansys ${ }^{\text {eeee }}$, International journal of engineering research and applications, 2(5) (2012) 2021-2027.

[37] R.L.N. . Vijaya Kumar1, Ch.Srinivas3, Design and Analysis of Gas Turbine Blade by Potential Flow Approach, Int. Journal of Engineering Research and .Application.www.ijera.com 4(1) (January-2014. ).

[38] B.H. Dennis, I.N. Egorov, G.S. Dulikravich, S. Yoshimura, Optimization of a large number of coolant passages located close to the surface of a turbine blade, in: ASME Turbo Expo 2003, collocated with the 2003 International Joint Power Generation Conference, American Society of Mechanical Engineers Digital Collection, 2003, pp. 13-19.

[39] G.S. Dulikravich, T.J. Martin, Z.-X. Han, Aero-thermal optimization of 
internally cooled turbine blades, Computational fluid dynamics'98, (1998) 158-161.

[40] S. Kennon, G. Dulikravich, The inverse design of internally cooled turbine blades, (1985).

[41] S. Amaral, T. Verstraete, R. Van den Braembussche, T. Arts, Design and optimization of the internal cooling channels of a high pressure turbine blade - part I: methodology, Journal of turbomachinery, 132(2) (2010).

[42] T. Verstraete, S. Amaral, R. Van den Braembussche, T. Arts, Design and optimization of the internal cooling channels of a high pressure turbine blade - part II: optimization, Journal of turbomachinery, 132(2) (2010).

[43] H. Namgoong, C. Son, P. Ireland, U-bend shaped turbine blade cooling passage optimization, in: 12th AIAA/ISSMO multidisciplinary analysis and optimization conference, 2008, pp. 5926.

[44] N. Nagaiah, Multiobjective design optimization of gas turbine blade with emphasis on internal cooling, (2012).

[45] G. Nowak, W. Wróblewski, I. Nowak, Convective cooling optimization of a blade for a supercritical steam turbine, International Journal of Heat and Mass Transfer, 55(17-18) (2012) 4511-4520. 